\title{
Enfermería de salud mental: El arte y la ciencia del cuidar
}

\author{
Mental Health Nursing: The Art and Science of Health Care
}

\section{María Teresa Lluch Canut}

Catedrática de Enfermería Psicosocial y Salud Mental.

Escuela de Enfermería. Facultad de Medicina y Ciencias de la Salud. Universidad de Barcelona.

Contacto: tlluch@ub.edu

Fecha de recepción: 5 de marzo de 2018 / Fecha de aceptación: 16 de mayo de 2018

Los profesionales de enfermería estamos trabajando, desde hace décadas, en la construcción de conocimiento científico y evidencia empírica para poder afirmar con robustez que la enfermería es una ciencia. Sin embargo, cuando a ello le unimos el arte, los cuidados de enfermería pasan a ser de un nivel superior. Interviene no sólo el conocimiento y el razonamiento crítico sino también la sensibilidad, el respeto y la proximidad hacía el ser humano en los momentos en que necesita ayuda.

Se hace difícil enmarcar los aspectos principales de cada apartado de un proceso de atención de enfermería sin contemplar los movimientos de sensibilidad específica que se dan en el sistema emocional de la persona. Es ese espacio donde todos deberíamos ubicarnos en momentos específicos de nuestro proceso existencial. Los profesionales de enfermería de salud mental estamos especialmente formados en respuestas emocionales del ser humano, en su comprensión y en el abordaje de las emociones.

Sin embargo, para manejar emociones se requiere una sensibilidad especial que es la que configura la parte artística de nuestra profesión. Son muchos los momentos en que hay que aplicar creatividad, iniciativa, ingenio y una visión amplia de la situación de cuidados, para poder dar la respuesta adecuada a las necesidades de los pacientes/usuarios y a sus familias.

La dualidad (arte y ciencia) forma parte de la esencia propia del cuidar. Cuando las personas tenemos problemas de salud mental y necesitamos la ayuda de un profesional, esperamos encontrar un profesional que proporcione soporte técnico y esté bien preparado científicamente (conocimientos), pero también que tenga sensibilidad y capacidad de comprensión para saber aplicar los conocimientos adaptados a las necesidades emocionales de cada persona y de cada situación.

Para estar bien preparado científicamente, es necesaria la formación continuada y la investigación. El concepto fundamental que desarrollar es el lifelong learning o formación a lo largo de toda la vida. Y manteniendo presente que la investigación es el instrumento fundamental para potenciar la parte científica del cuidar. Es necesario describir, correlacionar, confirmar, refutar teorías y modelos; construir y validar instrumentos de valoración; demostrar la efectividad de las intervenciones que realizamos; validar sistemas de medida de resultados, y buscar métodos de cuidados con equilibrio coste-efectividad. También es necesario formularse preguntas básicas: “¿Lo hago bien?: lo voy a demostrar, lo voy a compartir”; “¿Puedo hacerlo mejor?: lo voy a explorar”.

En este sentido, todo profesional de enfermería debe realizar investigación, en su quehacer profesional, intentando realizar una atención basada en cuidados efectivos, de calidad, que proporcionen el mayor grado de satisfacción posible tanto a los beneficiarios como a quien los administra, y sin obviar que todo proceso de cuidados también tiene un coste que hay que gestionar. Pero es evidente que no todos los profesionales de enfermería tienen el mismo nivel de formación en investigación. Y no todos han de desarrollar la competencia investigadora en el mismo grado de profundidad y amplitud. 
En este punto es necesario hablar del doctorado en enfermería, una formación académica que permite el desarrollo profesional de la capacidad investigadora en su máximo potencial, dirigiendo proyectos competitivos de investigación nacionales e internacionales y participando en comités de investigación de instituciones y servicios científicotécnicos de evaluación y acreditación nacional e internacional. Si bien no es necesario que todos los profesionales de enfermería se doctoren, el doctorado en enfermería es imprescindible. Actualmente, los centros sanitarios precisan tener enfermeros/as doctores/as para poder actuar de líderes en el desarrollo profesional de la investigación de los cuidados enfermeros. Ya que, tal y como acabamos de señalar, la investigación ha de ir inherente al cuidar. Sólo así se podrá avanzar y mejorar en la calidad del cuidar y en el desarrollo de nuestra profesión. Las doctoras y doctores en enfermería han de ayudar, acompañar, estimular, apoyar y asesorar a sus colegas en el desarrollo de la investigación ejerciendo un liderazgo con humildad, generosidad y saber estar, creando grupos de investigación y guiando proyectos que aporten conocimiento científico para el avance de la profesión y de los cuidados de calidad.

Sin embargo, la formación y la investigación por sí solas no garantizan la calidad del cuidar. Es necesario añadir el ingrediente artístico del cuidar. Cuidar es un arte. Ayudar a las personas cuando ellas no pueden hacerlo por sí solas es un arte. Y hay profesionales que tienen más arte que otros. ¿Cómo se puede potenciar nuestra capacidad artística para cuidar? Desde mi punto de vista, hay cuatro ingredientes, muy inherentes en la enfermería de salud mental:

El primer ingrediente consiste en impregnarse de la auténtica filosofía del cuidar. Cuando se define a la persona como un ser bio-psico-social-espiritual y cuando se dice que el cuidado que tenemos que hacer debe ser integral (holístico), esto nos lo tenemos que creer de verdad. Cuando estamos administrando una medicación, podemos al mismo tiempo tener palabras tranquilizadoras o regalarle una sonrisa. Esto se puede enseñar (y, de hecho, se enseña) pero sólo se puede aprender si de verdad nos lo creemos. "Si vemos a la persona en su globalidad".

El segundo ingrediente consiste en aplicar las actitudes terapéuticas básicas que todo profesional de enfermería aprende en la formación de grado y potencia en la formación especializada, destacando como más importantes el respeto, la escucha activa y la empatía. Ser respetuoso implica saber superar los propios prejuicios y aplicar la aceptación de las personas como tales (aunque no aceptamos sus comportamientos). Saber escuchar implica ser capaz de captar lo que la persona nos quiere decir (no lo que nosotros quisiéramos oír). Y empatizar implica tener nuestros canales emocionales abiertos (sensibilidad) y a la vez diferenciar bien entre nuestros propios sentimientos y los sentimientos de los demás. "El sufrimiento de los demás no nos puede dejar indiferentes, pero tampoco puede absorbernos tanto que se convierta en propio".

Un tercer ingrediente necesario para potenciar la vertiente artística del cuidado consiste en tener ganas de ayudar. Tener ganas de intentar hacerlo bien. Los conocimientos deben ir acompañados de las ganas de aplicarlos lo mejor posible. A veces nos faltan los recursos para poder hacer las cosas como nos gustaría, para poder ayudar como de verdad creemos que hay que hacerlo. Pero "habrá una gran diferencia entre lo que puede hacer un profesional que tenga ganas de ayudar y uno que no tenga".

El cuarto ingrediente necesario para potenciar la capacidad artística de los profesionales del cuidado consiste en saber cuidarse a sí mismo: autocuidado. Cuidarse para poder cuidar mejor. "Un artista del cuidar no puede dejar de cuidarse a sí mismo porque, en definitiva, debemos ser modelos de referencia para otras personas".

Para finalizar este editorial, quisiera señalar que soy consciente de que muchas de las cuestiones planteadas son absolutamente conocidas y aplicadas por los profesionales de la enfermería de salud mental. Justamente la especialidad de salud mental nos da a las enfermeras y enfermeros una dosis especial de formación en aspectos psicosociales y espirituales de los cuidados. Y es así como vinculamos la idea de que para cuidar es preciso utilizar de forma conjunta "la cabeza y el corazón". Y es sólo así que podremos desarrollar al máximo el arte y la ciencia del cuidar en la enfermería de salud mental. 